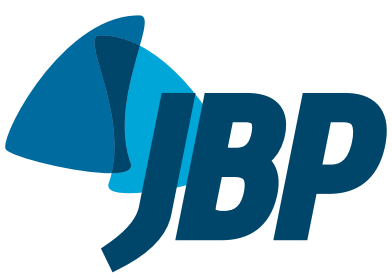

\title{
Pulmonary fat embolism of neoplastic origin
}

\author{
Flávia Pinto ${ }^{1}$, Miriam Menna Barreto ${ }^{1}$, Daniela Braz Parente ${ }^{1}$, Edson Marchiori ${ }^{1}$
}

\section{TO THE EDITOR:}

We report the case of a 67-year-old female patient who had hypertension and diabetes and had recently been diagnosed with a right renal mass. Abdominal CT and magnetic resonance imaging (MRI) showed that the renal mass, which measured approximately $7.5 \mathrm{~cm}$ along its longest axis, was predominantly exophytic, was located in the upper half of the right kidney, and had areas suggestive of intratumoral fat; in addition, the signal of the lesion extended to the ipsilateral renal vein and the inferior vena cava (Figure 1A). Histopathological examination of a CT-guided percutaneous biopsy sample revealed the presence of clear cells and oxyphilic cells that were probably malignant, with a moderately elevated proliferative index; a diagnosis of malignant primary renal neoplasm was therefore made.

Shortly thereafter, the patient presented to the emergency room with respiratory complaints. A CT scan of the chest revealed the presence of negative (fat) density in a branch of the left pulmonary artery (Figure 1B). A chest CT angiography was subsequently performed and confirmed the presence of pulmonary fat embolism (Figures 1C and 1D). Therefore, a diagnosis of pulmonary embolism secondary to renal cell carcinoma was made.

It is important to emphasize that the diagnosis of pulmonary tumor embolism was based on CT and MRI findings. Abdominal CT showed that the embolus had negative density values, which are characteristic of fat. Contrast-enhanced MRI of the thorax was also performed, showing a signal drop in the thrombus on the fat-saturated and out-of-phase T1-weighted images, thus confirming the presence of pulmonary fat embolism. The same was found for the renal tumor and its extensions to the ipsilateral renal vein and the inferior vena cava.

An imaging finding of fat in a renal tumor is highly suggestive of angiomyolipoma. Fat is rarely associated
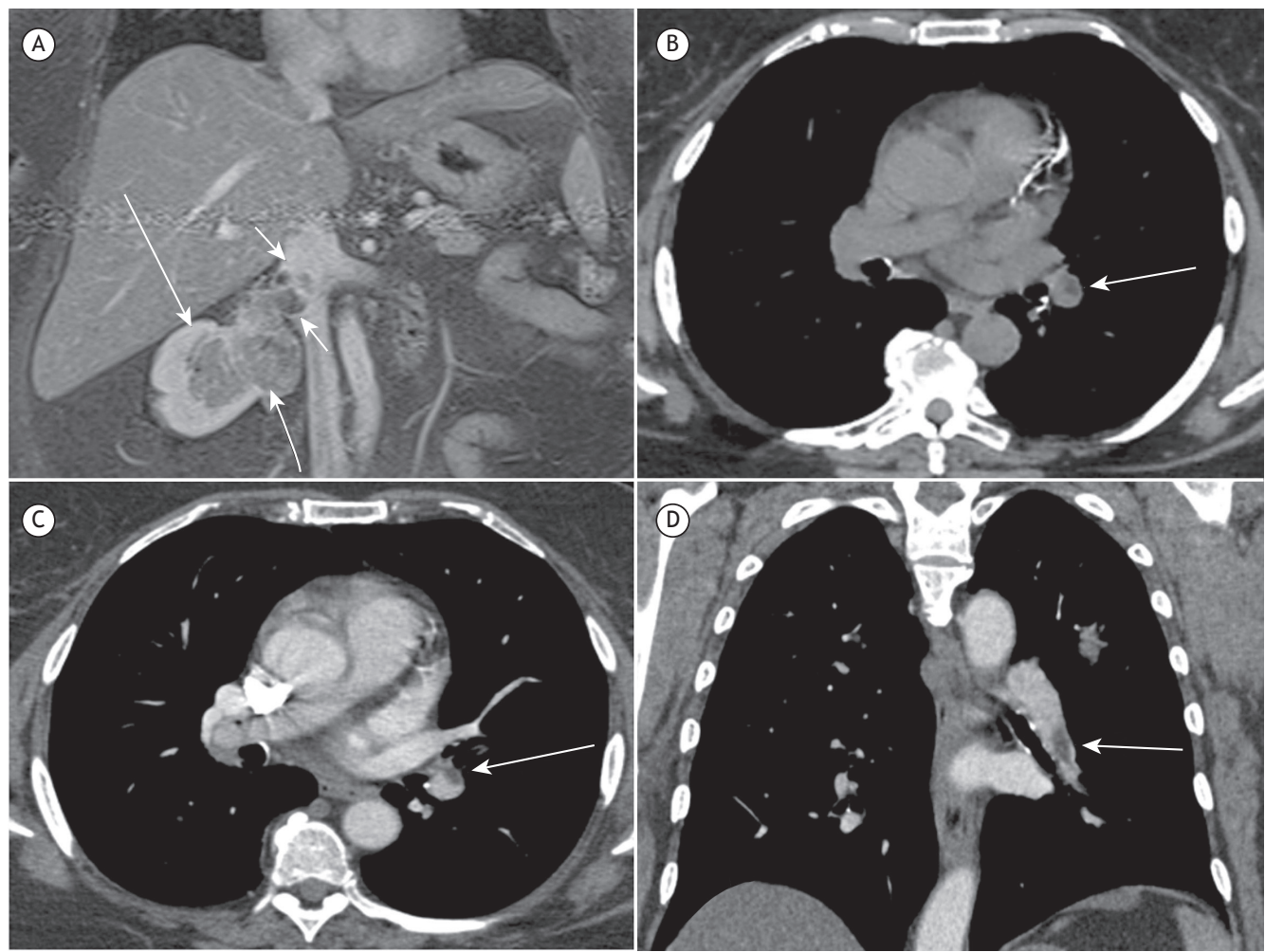

Figure 1. In A, coronal contrast-enhanced fat-saturated T1-weighted magnetic resonance image showing a renal tumor in the renal sinus (arrows), invading the right renal vein and extending into the inferior vena cava (arrowheads). In B, axial CT scan without contrast enhancement, showing a filling defect with fat density in a branch of the left pulmonary artery (arrow). Axial and coronal CT angiography scans of the chest in C and D, respectively, showing a fatty embolus partially obstructing the pulmonary artery branch to the left lower lobe (arrows). Density values for the tumor embolus ranged from -30 to -60 Hounsfield units. 
with other renal neoplasms, such as renal cell carcinomas. ${ }^{(1)}$ Cases of pulmonary embolism secondary to renal tumors have been reported in the literature, most of which were cases of pulmonary fat embolism secondary to angiomyolipoma. ${ }^{(2-4)}$ To our knowledge, there has been only one reported case of pulmonary embolism secondary to renal cell carcinoma, ${ }^{(5)}$ albeit without fatty emboli. Therefore, in addition to the known association between renal angiomyolipoma and fatty emboli in the pulmonary arteries, there is an association between renal cell carcinoma and fatty emboli, as demonstrated by the case reported herein.
Renal cell carcinoma is the most common type of kidney cancer, with metastatic disease at diagnosis in 20-25\% of cases. ${ }^{(5)}$ The association between renal tumors and pulmonary fat embolism is of note because, given the signal intensity and density characteristics on MRI and $\mathrm{CT}$, respectively-which are relatively specific to fat-a presumptive diagnosis can be made without contrast-enhanced imaging, as was the case here.

In conclusion, it is important to evaluate fat density thrombi in the pulmonary vessels of patients with renal neoplasms with or without respiratory symptoms for a diagnosis of pulmonary tumor embolism.

\section{REFERENCES}

1. Hammadeh MY, Thomas K, Philp T, Singh M. Renal cell carcinoma containing fat mimicking angiomyolipoma: demonstration with CT scan and histopathology. Eur Radiol. 1998;8(2):228-9. https://doi. org/10.1007/s003300050367

2. Harris K, Hatem E, Maroun R, Seminara D, Maniatis T. A renal angiomyolipoma with extension to the renal vein and asymptomatic fat pulmonary embolus. Ther Adv Respir Dis. 2014;8(4):133-5. https://doi.org/10.1177/1753465814538690

3. Hamidi N, Suer E, Gokce Ml, Alacayir I, Atasoy C, Kankaya D, et al.

An Unusual Manifestation of Renal Angiomyolipoma: Pulmonary Fat Embolism. Urology. 2015;86(3):e13-5. https://doi.org/10.1016/j. urology.2015.05.016

4. Yarmish G, DiPoce J. Case 199: Aggressive angiomyolipoma with renal vein thrombosis and pulmonary fat embolus. Radiology. 2013;269(2):615-8. https://doi.org/10.1148/radiol.13121187

5. Lim NK, Aik OT, Meng LL, Htun TH, Razack AH. Superior vena caval syndrome secondary to metastatic renal cell carcinoma. J Coll Physicians Surg Pak. 2014;24 Suppl 1:S68-70. 